\title{
SOBRE O CONCEITO DE COMPORTAMENTO: ALGUNS BREVES COMENTÁRIOS
}

\author{
ON THE CONCEPT OF BEHAVIOR: SOME BRIEF COMMENTS
}

FILIPE LAZZERI

UNIVERSIDADE DE SÃo PAULO, BRASIL

\begin{abstract}
Trabalho realizado a partir de pesquisa com apoio de bolsa de doutorado concedida ao autor pela Fundação de Amparo à Pesquisa do Estado de São Paulo (FAPESP), \#12/00059-2.

e-mail: filipelazzeri@gmail.com
\end{abstract}

\section{SOBRE O CONCEITO DE COMPORTAMENTO: ALGUNS BREVES COMENTÁRIOS}

Tive a tarefa de comentar, aqui, em um curto espaço, os artigos de Tonneau (2013), Todorov e Henriques (2013), Carrara e Zilio (2013) e Botomé (2013) publicados neste número especial da REBAC junto com um trabalho de minha autoria (a saber, Lazzeri 2013). Apresentarei alguns breves comentários a eles após ressaltar, resumidamente, algumas das teses que sustentei em meu referido trabalho.

Cada um dos artigos que aqui discuto tem seus méritos. Por uma questão de espaço, cingir-me-ei a certos aspectos desses artigos. Minhas ponderações sobre eles têm apenas um propósito construtivo e de contribuir para o tema em pauta. São ponderações que, se corretas, não afetam, de modo nenhum, todas as, mas apenas determinadas, ideias sugeridas neles, mesmo porque eu concordo com algumas de suas ideias.

\section{O QUE COMPORTAMENTO É E O QUE ELE NÃO É}

Em Lazzeri (2013), começo fazendo uma distinção entre diferentes sentidos do conceito de comportamento. Apontei quatro sentidos dele, nomeadamente: (i) comportamento como a ocorrência de uma ação ou reação de um organismo; (ii) comportamento como um padrão ou uma classe (de comportamentos na acepção i); (iii) comportamento de grupo; e (iv) comportamento como um movimento ou mudança de um objeto (ver também Lazzeri 2014a, 2014b). Há ainda pelo menos um quinto sentido do conceito, a saber: (v) comportamento como o repertório ou conjunto amplo de comportamentos no sentido de (ii) - isto é, conjunto de padrões ou classes de comportamento - de um organismo. Meu foco em Lazzeri (2013) é na acepção (i).

Além disso, ao longo daquele trabalho, eu ofereci, por meio de análise conceitual e com base em algumas contribuições anteriores, argumentos nos quais sustento que comportamento no sentido de (i) possui feições tais como, dentre outras, as seguintes: (a) sua extensão engloba desde certas coisas que um organismo realiza de modo relativamente automatizado e rígido - incluindo ocorrências, por exemplo, de cadeias de reações (reaction chains) e de padrões modais (ou fixos) de ação -; até coisas que realiza de modo relativamente flexível e espontâneo (em geral, ocorrências de padrões operantes); (b) trata-se de algo que contrasta com estados; (c) é algo que possui funções; (d) contrasta com pelo menos muitas dos processos desenvolvimentais do organismo; (e) não equivale a relações, embora seja algo que fundamentalmente possui propriedades relacionais; etc.

Distingui também entre os tipos de definição possivelmente adequados e aqueles que em geral não o são para o conceito e para o contexto relevantes. Por exemplo, apontei que comportamento, na acepção relevante naquele trabalho, isto é, na acepção (i), não corresponde a um conceito de similaridades de família, portanto não lhe cabendo uma definição disjuntiva de suficiência; mas que definições disjuntivas que (diferentemente das de suficiência) admitam a inclusão de condições necessárias relevantes podem ser de interesse.

Delimitei cinco desideratos para uma definição de comportamento na acepção relevante, para qualquer um dos tipos de definição que identifiquei como possivelmente adequados. Esses desideratos são: a definição (I) não deve ser demasiado restritiva; (II) não deve ser demasiado ampla; (III) não deve ser circular (salvo se a circularidade não for viciosa); (IV) deve ser informativa; e (V) deve, em geral, capturar as nuanças básicas do conceito, ou seja, nuanças tais como (a)-(e), que procurei explicitar ao longo do trabalho (ver também Lazzeri 2014b). Outro desiderato ainda - mas que, diferentemente daqueles, eu adotei apenas implicitamente (em especial, em meu exame da definição de 
Levitis et al. 2009) -, é o de que (VI) a definição não deve ser ad hoc.

Com base nessas análises e distinções, eu examinei doze definições de comportamento no sentido de (i) presentes na literatura científica e filosófica. Argumentei que elas enfrentam, de diferentes maneiras, dificuldades conceituais. E argumentei em favor de duas definições alternativas, que podem ser consideradas modificações daquelas que se encontram em Millikan (1993) e Moore (2008). Minhas definições - as quais podem eventualmente ser aperfeiçoadas - são, expressando-as sucintamente, as seguintes:

(D.1) Comportamento é aquilo que um organismo faz que possui uma ou mais funções (que devem ser entendidas, resumidamente falando, em termos de histórias de seleção filogenética ou ontogenética) e cuja etiologia envolve parcialmente fatores do ambiente presente como estímulos sensoriais [exteroceptivos, interoceptivos ou proprioceptivos]. (Lazzeri, 2013, p. 63)

(D.2) Comportamento é aquilo que um organismo faz cuja etiologia envolve (em parte) processos seletivos na filogênese ou na ontogênese do organismo e (em parte) fatores do ambiente presente atuando como estímulos sensoriais [exteroceptivos, interoceptivos ou proprioceptivos]. (Lazzeri, 2013, p.63)

As definições (D.1) e (D.2) são aproximadamente equivalentes. A diferença entre elas reside no fato de que, em (D.1), faço uso da terminologia de funções (ou propósitos), ao passo que, em (D.2), não, substituindo-a pela de histórias de seleção filogenéticas elou ontogenéticas do organismo. Em (D.1), funções são entendidas em termos desses processos históricos. 1

\section{RESPOSTAS DOS ORGANISMOS COMO COMPORTAMENTOS NA ACEPÇÃO (I)}

Todorov e Henriques (2013) sugerem que o termo 'resposta', em análise do comportamento, não é sinônimo de 'comportamento'. Segundo eles, "comportamento é classe, resposta é instância" (p. 76).

As respostas de um organismo são, certamente, instâncias (ou ocorrências), contrastando com classes (ou padrões) de respostas. Elas são coisas irrepetíveis e exemplificam ou compõem classes ou padrões ao longo do tempo. Porém, conforme elucido em Lazzeri (2013; ver também Lazzeri 2014a, 2014b), há um sentido do conceito de comportamento - a saber, o sentido (i) - que coincide

1 Penso que essas definições estão em larga consonância com a maneira como Skinner (e.g., 1953) pensa os comportamentos. Skinner tende a rejeitar a fala em termos de funções ou propósitos, mas a teoria etiológica das funções, como desenvolvida por Wright (1976) e Millikan (1984), é compatível com sua abordagem. aproximadamente com o conceito de resposta (mesmo como este último é utilizado em análise do comportamento). Comportamento como uma classe é apenas uma das acepções do conceito - a saber, a acepção aqui convencionalmente apontada como (ii). Respostas não são comportamentos na acepção (ii), mas são comportamentos na acepção (i). Inclusive, penso que há usos do conceito por analistas do comportamento equivalendo-o ao de resposta. Não há problema com esses usos, desde que os sentidos do conceito não sejam confundidos (já que isso pode gerar outras confusões conceituais). Utilizar o termo 'resposta' pode ajudar a distinguir qual é o sentido do conceito do qual se estiver falando.

\section{HÁ COMPORTAMENTOS (QUER NA ACEPÇÃO I OU NA ACEPÇÃO II) QUE NÃO SÃO DE TIPO OPERANTE}

Concordo com Carrara e Zilio (2013) em pontos como, por exemplo, os seguintes: a adequação de uma definição varia conforme, por exemplo, as finalidades que se tenha em vista com ela (eu fiz várias distinções relacionadas a isso em Lazzeri 2013, 2014b); o conceito de comportamento figura - eu ressaltaria, em diferentes sentidos do conceito - em diversas ciências (incluindo a física e a química, nas quais o sentido iv é o mais relevante); e ele - eu diria, na acepção (i) - diz respeito a (certas) coisas, mas não a qualquer coisa, que o organismo faz; 2 e, além disso, como recentemente parece apontar Todorov (2012), não corresponde a, ainda que envolva, relações.

Com Botomé (2013), concordo, por exemplo, que padrões operantes de comportamento podem, a princípio, ser razão em parte de consequências estendidas no tempo, e não apenas por consequências contíguas temporalmente. Acredito que (como já se prefigura em Skinner 1935), na caracterização dos operantes, não é conceitualmente necessário que haja contiguidade temporal entre as consequências das respostas e estas, mas sim que a consequência tenha uma influência na (isto é, aumente ou diminua a) probabilidade de ocorrência destas. Como salienta Botomé, trata-se de uma questão empírica se há, de fato, a influência de consequências de médio e longo prazos (embora, é claro, não do futuro para trás, devendo ser analisadas historicamente). Aparentemente, tal influência ocorre algumas vezes. A teoria do autocontrole de Rachlin (2000), por exemplo, baseia-se nessa premissa e, conforme indica Rachlin, há evidências experimentais em favor dela (embora haja aspectos falhos, a meu ver, na filosofia da mente de Rachlin).

No entanto, o texto de Carrara e Zilio (2013) e o de

2 Há, no entanto, um momento do texto de Carrara e Zilio (2013) em que eles dizem que "o que o organismo faz é comportamento" (p. 15). Isso parece ser inconsistente com o que eles afirmam anteriormente no texto; mas pode ser interpretado como uma simples maneira abreviada de falar resguardada a ressalva. 
Botomé (2013), em certo sentido, cometem uma pequena falha quanto ao desiderato (I) acima indicado, isto é, o desiderato de não ser demasiado restritivo 3. Carrara e Zilio (2013), por exemplo, dizem: "Se [comportamento] é, portanto, organismo funcionando ('fazendo'), o que é afetado (selecionado) é uma configuração específica de seu modo de fazer que implica um procedimento de reforçamento positivo ou negativo...." (p. 15). O problema com essa asserção é que há (conforme argumento em Lazzeri 2013, 2014b) comportamentos - quer se tome o conceito na acepção (i) ou, mutatis mutandis, na acepção (ii) - que não são oriundos ou necessariamente influenciados por processos de seleção via mecanismo de reforçamento. Esse é o caso, por exemplo, de ocorrências de cadeias de reação (reaction chains), de padrões modais (ou fixos) de ação e de taxias (taxes). Ou seja, há comportamentos - em qualquer uma das acepções relevantes - que não são de tipo operante. 4 Creio que esses autores reconheceriam isso, mas não o levaram suficientemente em conta nos referidos trabalhos.

Carrara e Zilio (2013) e Botomé (2013) declaram ter, nesses trabalhos, preocupação com o conceito de comportamento no âmbito específico da análise experimental do comportamento. Entretanto, a meu ver, isso não justifica a restrição deles praticamente a comportamentos de tipo operante. Isso porque, em primeiro lugar, eles não fazem, nesses trabalhos, asserções apenas sobre comportamentos operantes, com a qualificação de que são apenas os operantes, mas também sobre comportamentos (em geral), sem qualificação de que sejam apenas os de tipo operante. Ou seja, sua pretensão, em alguns momentos dos respectivos textos, não parece cingirse a comportamentos operantes.

Em segundo lugar, como sabemos, a análise experimental do comportamento reconhece que há comportamentos que não são de tipo operante. Alguns autores na área (e.g., Mazur 2006; Pierce \& Cheney 2004) reconhecem vários tipos de padrões de comportamento, tais como os que já mencionei (e.g., padrões modais de ação, taxias). Sabemos que a área considera efetivamente (inclusive há investigações nela sobre) os reflexos (ou respondentes).5 A meu ver, deve também considerar

3 Digo "em certo sentido" porque estritamente falando, eles não propõem, entretanto, definições da noção alvo, mas apenas alguns elementos que comporiam uma tal definição.

4 Alguns comportamentos desses tipos são, é claro, influenciados por mecanismos de reforço (e.g., podem ser otimizados pelo contato com contingências do habitat). Minha observação é compatível com isso, pois apenas procura chamar atenção para o fato de que, presumivelmente, nem todo comportamento advém ou é mantido por reforço.

5 Falo isso ciente de que a distinção entre operantes e reflexos (ou respondentes), porém, é problematizada por alguns autores na área; notadamente por Donahoe e Palmer (1994). efetivamente (para além de apenas reconhecer) esses outros tipos de comportamentos de base fortemente filogenética, de maneira a interagir mais com estudos etológicos e afins que sejam de modo amplo consistentes com ela. É verdade, a área incube-se preponderantemente dos operantes; mas, sendo parte de uma ciência maior do comportamento (Skinner 1990) e levando-se em conta que há interação entre operantes e outros tipos de comportamento, esses também merecem atenção por ela. 6

Para Carrara e Zilio (2013), "eventos da economia corporal interna", com "funções básicas de sobrevivência filogeneticamente estabelecidas" e que não são coisas "afetada[s] por consequências do ambiente externo da mesma forma em que o[s] operante[s]" (p. 15), não contam como comportamento. Carrara e Zilio (2013) têm em vista eventos como a circulação do sangue e a respiração regulares. Eu concordo que a circulação do sangue e a respiração regulares não são, a princípio, comportamentos em qualquer sentido relevante aqui (isto é, i ou ii). Porém, tal afirmação de Carrara e Zilio (2013) parece implicar algo mais geral; a saber, que inclusive muitos reflexos, como, por exemplo, o de salivação de um cão dado certos alimentos como estímulos sensoriais (e.g., gustatórios, olfativos) incondicionados, não contam como comportamento (por se tratar de eventos da economia corporal interna etc).

A meu ver, não há por que excluir da extensão do conceito reflexos como esse. Ocorrências deles exibem características gerais que as de operantes exibem, como as de não serem coisas que o organismo faz por inteira força de objetos do ambiente externo presente; não corresponderem à produção de meros resultados incidentais; e sua etiologia envolver parcialmente estímulos sensoriais do ambiente presente. Considero que esses fenômenos contam, mas que circulação sanguínea e respiração regulares não contam, como extensão do conceito (em acepção relevante), pelo fato de que aqueles, mas não estes, são coisas que um organismo faz que possuem funções e que são parcialmente causadas por fatores do ambiente antecedente atuando como estímulos sensoriais.

Falo em circulação sanguínea e respiração 'regulares' para contrastar com os casos em que correspondem a reflexos condicionados, os quais considero que contam como comportamentos. Respirar profundamente e aumentar o batimento cardíaco dada a presença de um som associado, por condicionamento pavloviano, à presença de um predador, por exemplo, contam como comportamentos em minha proposta (ver Lazzeri 2013). Não ficou claro para mim se Carrara e Zilio (2013) considerariam esses casos como comportamentos (em acepção relevante). Por um lado,

6 Pode-se tentar definir os padrões modais (ou fixos) de ação, taxias e similares como subtipos de reflexos. Isso pode ser bastante plausível. Em todo caso, dificilmente poder-se-ia dizer que todos eles envolvem (necessariamente) mecanismo de reforço. 
conotam considerar que o 'respirar ofegante' conta na extensão do conceito; mas, por outro lado, reflexos condicionados, como tipicamente são entendidos na literatura, não necessariamente envolvem reforço (ou seleção operante).

\section{OS QUATRO CRITÉRIOS DE TONNEAU (2013) (COMO ELE PRÓPRIO CONOTA) NÃO SÃO CONDIÇÕES CONJUNTAMENTE SUFICIENTES DE COMPORTAMENTO (NA ACEPÇÃO I)}

Tonneau (2013) sugere que comportamentos - e ele parece ter em vista o que aponto como acepção (i) do conceito - são ocorrências que satisfazem pelo menos os seguintes critérios: (1) trata-se de ocorrências que envolvem um efeito nos limites ou fronteiras do organismo; (2) esse efeito é resultante de atividade interna do organismo; (3) a relação causal entre a atividade e o efeito envolve uma função biológica (que Tonneau 2013 parece entender em termos de ter origem fundamentalmente via seleção natural); e (4) o efeito é reversível (em um sentido de reversível que não ficou claro para mim). Tonneau (2013) não supõe que esses quatro critérios sejam conjuntamente suficientes, mas apenas que são condições necessárias, para algo contar como comportamento (na acepção relevante).

De fato, se esses critérios fossem tomados como condições conjuntamente suficientes de comportamento (na acepção relevante), então ter-se-ia, acredito, uma definição demasiado abrangente, isto é, que falharia quanto ao desiderato (II) acima referido. Pois esses quatro critérios não são capazes, por si sós, de excluírem da extensão do conceito (na acepção relevante) coisas que correspondem à produção pelo organismo de resultados meramente incidentais. Por exemplo, uma pessoa, ao movimentar um braço, produzir sombra de modo incidental sobre uma formiga que acontece de estar próxima, não é uma ação ou reação da pessoa (supondo que a consequência produzida seja meramente por acaso). Mas essa produção da sombra sobre a formiga é algo que envolve movimentação do braço pela pessoa (o que satisfaz o critério 1 do autor), resultante de uma atividade interna muscular (o que satisfaz o critério 2 do autor) que em última instância envolve mecanismos causais advindos (pelo menos em parte) por seleção natural (o que satisfaz o critério 3 do autor). E, ademais, o autor consideraria esse movimento do braço um efeito reversível (o que satisfaz seu critério 4). Como ele explicitamente sugere, os movimentos dos animais em geral, desde que envolvendo causas internas (ficando de fora, por exemplo, os meros movimentos do organismo em torno do eixo terrestre), contam como comportamentos (na acepção relevante). Logo, se os quatro critérios de Tonneau (2013) fossem considerados como uma definição, múltiplas coisas que os organismos fazem correspondentes à produção de resultados meramente incidentais seriam contados, a partir dela, como itens da extensão do conceito, o que seria um erro conceitual grave (ver Lazzeri 2013, 2014a, 2014b).

Essa minha observação, se correta, sinaliza para uma limitação dos critérios de Tonneau (2013). Seus critérios têm o mérito de excluir da extensão do conceito coisas que o organismo faz por simples força do ambiente externo presente (e.g., um braço ou pata de um organismo mover-se por inteira força de outrem) e, aparentemente, várias atividades desenvolvimentais (e.g., formação dos dedos em mamíferos), já que (como argumento em Lazzeri 2013, 2014b) o conceito envolve tais contrastes. A limitação dos critérios de Tonneau (2013), se minha observação estiver correta, é o fato de elas não excluírem os casos de produção pelo organismo de meros resultados incidentais.

As definições que sugiro (Lazzeri, 2013), por outro lado, excluem, efetivamente, tanto coisas que o organismo faz por inteira força do ambiente externo imediato e pelo menos várias atividades desenvolvimentais, como também os casos de produção de resultados incidentais.

\section{SERÃO OS MOVIMENTOS NÃO ACIDENTAIS DE UM ORGANISMO EM GERAL COMPORTAMENTOS (NA ACEPÇÃO I)?}

Tonneau (2013) afirma que o movimento animal, mesmo o de partes do organismo, conta como comportamento (na acepção relevante, que é a i). Suponho que o autor tenha em vista aqueles movimentos que não são meramente acidentais, como o é um movimento da pata de um animal ao ser movida forçadamente por outrem. Com efeito, parece seguir-se dos quatro critérios sugeridos por Tonneau (2013) para delimitar o conceito (na acepção relevante) que os movimentos não acidentais dos animais são em geral itens de sua extensão.

No entanto, se assim fosse, então, em uma corrida, por exemplo, os vários movimentos executados por diferentes partes do corpo de um animal (no caso de um ser humano, cada movimento dos braços, cotovelos, pernas, pés, dedos, cabeça, etc.) contariam como comportamento, conquanto envolvessem, grosso modo, causas internas ao corpo funcionando em razão de mecanismos moldados (pelo menos em parte) por seleção natural.

Eu discordo a esse respeito, pois penso que muitos desses movimentos não têm nenhum propósito ou função (apesar de terem subjacentes mecanismos fisiológicos que têm certas funções). Dito simplificadamente, alguns desses movimentos não são controlados por suas consequências e não são produzidos por mecanismos fisiológicos com função de produzi-los dados certos estímulos sensoriais. Nos casos em que os movimentos não possuem essas características, não possuem função (cf. Artiga 2010; Millikan 1984).

\section{NÃO HAVERÁ COMPORTAMENTOS (NA ACEPÇÃO I) QUE NÃO ENVOLVEM EFEITOS NAS FRONTEIRAS CORPÓREAS DO ORGANISMO?}

Tonneau (2013) sugere que todo comportamento 
(na acepção relevante) envolve um efeito nos limites ou fronteiras do organismo. Eu tenho dúvida sobre a plausibilidade desse critério. Não é claro se coisas como, por exemplo, secreções endócrinas (eliciadas por estímulos quer incondicionados ou condicionados) envolvam necessariamente esse tipo de efeito. Além disso, quando uma pessoa, por exemplo, ao ver (digamos) uma obra de Aristóteles em sua frente, lembra-se de um professor com quem teve aulas sobre textos de Aristóteles, há, presumivelmente, uma ou mais ocorrências de comportamentos reflexos envolvidos nesse lembrar-se particular, eliciados pelo livro em sua frente funcionando como estímulo eliciador condicionado. Não é claro se esses comportamentos necessariamente envolvem efeitos nas fronteiras do organismo. Eles podem envolver esse tipo de efeito em vários casos, mas o envolvem sempre?

Acredito que Tonneau (2013) consideraria que tais casos sempre envolvem efeito nas fronteiras do organismo. Ele considera que até mesmo "atividades neurais silenciosas" o envolvem (não querendo eu com isso, porém, conotar que o caso de reflexos envolvidos no lembrar-se mencionado antes simplesmente identifiquem-se com atividades neurais). Mas, nesse caso, o que o critério de que comportamento (em sentido relevante) deve envolver esse tipo de efeito exclui distintivamente, não excluído já pela fala no segundo critério de Tonneau (2013) em termos de atividade interna do organismo? Trata-se de uma dúvida.

\section{COMPORTAMENTO (NA ACEPÇÃO I) É, EM PARTE, ALGO QUE O ORGANISMO FAZ (MAS NÃO QUALQUER COISA QUE ELE FAZ)}

Como apontei em Lazzeri (2013, 2014a), mencionando Millikan (1993), comportamentos, na acepção (i), não equivalem a tudo que um organismo faz. Pois cair, resvalar, atrair a atenção de um predador, etc. são coisas que um organismo pode fazer incidentalmente ou por inteira força de eventos externos do ambiente imediato.

Por isso, a afirmação de Todorov e Henriques (2013) de que "os verbos e expressões que designam o que um indivíduo fez, faz ou fará são genericamente denominados [sic.] comportamentos" (p. 75) é problemática. Todorov e Henriques (2013) declaram ter foco em comportamento como classe de respostas (isto é, na acepção ii), ao invés de comportamento na acepção (i); mas é natural se entender nessa afirmação o termo 'comportamentos' no sentido de (i), já que uma classe de respostas não é o tipo de coisa que um organismo faça.

Tonneau (2013), como eu, alude ao fato de que nem tudo que um organismo faz é comportamento (novamente, acredito que Tonneau tenha em vista a acepção i), mencionando Millikan. Curiosamente, porém, Tonneau (2013) parece sugerir, ao mesmo tempo, que falar em o que um organismo faz não difere de falar em 'comportamento' no sentido relevante. Pois parece sugerir que, ao se introduzir aquela expressão no definiens, incorre-se em circularidade. Trata-se de dois pontos mutuamente inconsistentes. Ou sustenta-se que comportamento (em sentido relevante) não é qualquer coisa que um organismo faz, ou então que o que um organismo faz é sinônimo de comportamento (no sentido relevante).

Comportamento (na acepção relevante), a meu ver, não é qualquer coisa que um organismo faz, mas é em parte algo que o organismo faz. Penso, portanto, que o uso da expressão 'o que um organismo faz' ou de similar no definiens não necessariamente incorre em circularidade. A expressão 'o que um organismo faz' é, de modo aproximado, equivalente à expressão 'mudança do/pelo organismo', podendo, em geral, ser intersubstituídas.

\section{SOBRE O STATUS DOS COMPORTAMENTOS DE GRUPOS}

Por fim, eu tecerei um comentário sobre o status dos comportamentos de grupos, isto é, aqueles no sentido de (iii). Carrara e Zilio (2013, ao final do texto) parecem sugerir que, estritamente falando, apenas organismos singulares se comportam. Eles afirmam (citando Skinner 1953) que "é sempre o indivíduo que se comporta" e, além disso, que "não há consequência para o grupo" (p. 15). Para Carrara e Zilio (2013), então, aparentemente, comportamento de grupo é apenas uma maneira metafórica de falar de um fenômeno complexo que se reduz ontologicamente à soma dos comportamentos em sentido (i) de dois ou mais organismos.

Eu, porém, penso que comportamentos de grupos possuem propriedades que os comportamentos em sentido (i) que os compõem não possuem. Penso que há consequências produzidas pelo grupo como um todo e que isso é algo distintivo dos comportamentos de grupo. No entanto, concordo que coisas como "instinto de rebanho" e "caráter nacional", mencionados por Carrara e Zilio (2013), provavelmente são, de fato, meras façons de parler, e não reais comportamentos. $\mathrm{O}$ que considero como comportamentos de grupo são aqueles fenômenos (como, e.g., a caça de gnus por leões em conjunto) que envolvem dois ou mais organismos realizando comportamentos em sentido (i) em coordenação (ou sincronia) de maneira cooperativa, de tal modo que a consequência que produzem resulta dessa coordenação, por oposição a ser uma consequência produzida pelos organismos envolvidos tomados isoladamente (cf., e.g., Searle 2002/1990).

Tome-se, por exemplo, o comportamento de remar de um grupo, em direção a um local distante. Se o barco em que o grupo está é remado apenas em um dos lados, não segue em frente, mas, antes, gira para o lado oposto. Suponhamos que o grupo seja formado por duas pessoas, uma encarregada do remo da esquerda e, a outra, do remo da direita; e que cada uma delas cumpre sua tarefa. O comportamento desse grupo é composto por 
comportamentos individuais de remar realizados pelos organismos singulares que o formam, mas a consequência saliente produzida - a saber, a de mover o barco para $o$ destino alvo - resulta, neste caso, dos esforços sincronizados do grupo como um todo.7 Ou seja, ela é produzida pelo grupo como um todo, por contraste a ser uma consequência dos comportamentos individuais tomados isoladamente.

\section{REFERÊNCIAS}

Artiga, M. (2010). Learning and selection processes. Theoria, 25, 197-208.

Botomé, S. P. (2013). O conceito de comportamento operante como problema. Revista Brasileira de Análise do Comportamento, 9(1), 19-46.

Carrara, K., \& Zilio, D. (2013). O comportamento diante do paradigma behaviorista radical. Revista Brasileira de Análise do Comportamento, 9(1), 1-18.

Donahoe, J. W., \& Palmer, D. C. (1994). Learning and complex behavior. Boston: Allyn and Bacon.

Lazzeri, F. (2013). Um estudo sobre definições de comportamento. Revista Brasileira de Análise do Comportamento, 9(1), 47-65.

Lazzeri, F. (2014a). A conceptual difficulty with some definitions of behavior. Em J. Conte \& C. A. Mortari (Eds.), Temas em filosofia contemporânea (pp.148-155). Florianópolis: NEL/UFSC.

Lazzeri, F. (2014b). On defining behavior: Some notes. Behavior and Philosophy, 41, 65-82.

Levitis, D. A., Lidicker Jr., W. Z., \& Freund, G. (2009). Behavioural biologists do not agree on what constitutes behaviour. Animal Behaviour, 78, 103-110.

Mazur, J. E. (2006). Learning and behavior (6 ${ }^{\text {th }}$ ed.). Upper Saddle River, NJ: Prentice Hall.

Millikan, R. G. (1984). Language, thought, and other biological categories: New foundations for realism. Cambridge: MIT Press.

Millikan, R. G. (1993). What is behavior? A philosophical essay on ethology and individualism in psychology, part 1. In White queen psychology and other essays for Alice (pp.135-150). Cambridge: MIT Press.

Moore, J. (2008). Conceptual foundations of radical behaviorism. Cornwall-on-Hudson: Sloan.

$7 \mathrm{O}$ barco poderia seguir em frente por meio do esforço de apenas uma dessas pessoas que manejasse adequadamente ambos os remos. Porém, se essa fosse a situação, não mais faria sentido falar em ocorrência nela de um comportamento de remar de um grupo, sequer metaforicamente.
Pierce, W. D., \& Cheney, C. D. (2004). Behavior analysis and learning ( $3^{\text {rd }}$ ed.). Mahwah: Lawrence Erlbaum.

Rachlin, H. (2004). The science of self-control. Cambridge: Harvard University Press.

Searle, J. R. (2002/1990). Collective intentions and actions. Em Consciousness and language (pp.90-105). Cambridge: Cambridge University Press.

Skinner, B. F. (1935). The generic nature of the concepts of stimulus and response. Journal of General Psychology, $12,40-65$.

Skinner, B. F. (1953). Science and human behavior. New York: Macmillan.

Skinner, B. F. (1990). Can psychology be a science of mind? American Psychologist, 45, 1206-1210.

Todorov, J. C. (2012). Sobre uma definição de comportamento. Perspectivas em Análise do Comportamento, 3, 32-37.

Todorov, J. C., \& Henriques, M. B. (2013). O que não é e o que poderia vir a ser comportamento". Revista Brasileira de Análise do Comportamento, 9(1), 74-78.

Tonneau, F. (2013). Behavior and the skin. Revista Brasileira de Análise do Comportamento, 9(1), 66-73.

Wright, L. (1976). Teleological explanations: An etiological analysis of goals and functions. Berkeley: University of California Press. 